\title{
MATRIMONIO Y MODO DE VIDA DE UNA FAMILIA DE LA PEQUEÑA NOBLEZA PERIFERICA: LOS BOURGUNYO DE ALICANTE
}

\author{
Verónica MATEO RIPOLL \\ Universidad de Alicante.
}

El estudio sobre la familia es uno de los campos de investigación donde con mayor impulso renovador se ha trabajado en los últimos años. Dentro del amplísimo abanico de cuestiones que abarca, ha llamado poderosamente la atención la conexión existente entre matrimonio y patrimonio; dos realidades que, estrechamente unidas, formaron uno de los ejes de la vertebración de los grupos de poder durante la Edad Moderna (1). Es precisamente en esta práctica de historiar familias donde hemos situado nuestra investigación, con el fin de contribuir al conocimiento de la élite de poder de la sociedad alicantina del Setecientos. La familia Bourgunyo, objeto de este trabajo, despuntó como una de las más representativas de la oligarquía de caballeros y ciudadanos del municipio. El estudio de sus pautas de comportamiento, prototípicas y fuertemente ilustradoras de su status, puede servirnos como modelo para aventurar una fisonomía de la pequeña nobleza alicantina en este período.

El análisis de las estrategias matrimoniales es una de las vías más interesantes para conocer la posición exacta de una familia en la escala social, ya que supone un medio eficaz para avanzar en sus ambiciones. En este sentido, si atendemos al cuadro genealógico adjunto (2), observaremos el marcado carácter endogámico -tanto local como social- que existía en las alianzas de la Casa de Bourgunyo. Sin embargo, al tratarse de uno de los linajes locales de mayor solera y raigambre, no se valió de este medio para ascender en la escala social sino que fue utilizado para asegurar su posición y prestigio. Así, un estudio en profundidad de sus enlaces, recogidos en el cuadro I, nos permite obtener una serie de rasgos comunes: 


\section{CUADRO I}

CONTRAYENTES

\section{FECHA de la UNION}

1650

1713

1744

1765

1785

FUENTE: A.H.N Estado. Indice de pruebas de Carlos III. Expediente 460. Elaboración propia.

En primer lugar los nexos enunciados suponen, en todos los casos, el entroncamiento con estirpes que habían obtenido el privilegio de nobleza antes del año 1695. En lo que respecta a los Bourgunyo, las crónicas y nobiliarios locales nos informan de su origen catalán -y subsidiariamente francés- y de su posterior llegada al Reino de Valencia con el grupo de los caballeros que acompañaron al rey Jaime en la conquista (3). Su pertenencia, por lo tanto a la nobleza "de sangre y solar" y su tradición de prosapia militar y de caballeros, fueron algunos de los méritos que inclinaron a la Real Audiencia de Valencia a dictar, el 15 de septiembre de 1695, la sentencia probatoria de su nobleza (4). Pero casi un siglo antes, concretamente en 1581, los Remiro Alvarez de Espejo, habían conseguido despuntar como la primera familia alicantina que obtenía tal mención, mientras que los linajes de los Canicia y los Juan lo harían en 1589 y 1675 respectivamente (5).

Por otra parte, las uniones se realizan entre estirpes que ostentaron el poder municipal tanto en época foral como tras las reformas borbónicas. Los Juan ejercieron el cargo de Jurado en más de una veintena de ocasiones durante el siglo XVII; mientras que los Canicia demostraron sobradamente su apego a la política hasta los primeros años del siglo XIX. La presencia de los Bourgunyo en los órganos rectores municipales comenzó a mediados del siglo XV, aunque algunos nobiliarios consultados remontan esta fecha hasta el año 1372, cuando, al perecer, Gines Bourgunyo fue elegido Justicia (6). Pero dejando al margen esta cuestión, por otra parte imposible de documentar (7), su actividad política en el municipio fue continua desde la implantación del sistema insaculatorio hasta 1814. Durante el siglo XV disfrutaron en ocho ocasiones del ejercicio de la máxima autoridad y otras tantas del cargo de Jurado. En la siguiente centuria se observa un significativo descenso en el número de años que fueron Justicias; pues sólo lo fueron en 1501 y 1589 , aunque fue en el cumplimiento del oficio de Jurado donde tuvieron una mayor presencia. A lo largo del periodo foral hemos documentado el cargo de Jurat en manos de la familia Bourgunyo en veinticuatro oca- 
siones algunas de las cuales en años sucesivos, a pesar de estar prohibido por las ordenanzas (8); sólo en el siglo XVII su actividad política se ampliará al ejecutar, además de las citadas, las tareas propias de síndico y clavario (9).

Como se ha puesto de manifiesto en muchas ocasiones, los cambios impuestos en el territorio valenciano tras los Decretos de Nueva Planta afectaron escasamente a las familias que tradicionalmente habían ostentando los resortes del poder local. Es por ello que encontraremos a los Bourgunyo en la nueva administración municipal borbónica, actuando desde las regidurías del estado noble de forma ininterrumpida hasta el año 1814.

Cuando tiene lugar la formación del primer Ayuntamiento en 1709, Pedro Bourgunyo y Remiro y Antonio Rotla y Canicia, en atención a la fidelidad demostrada y sus destacadas actuaciones en la defensa de la Plaza durante la guerra de Sucesión, fueron designados regidor decano y segundo regidor respectivamente (10). Tras la muerte de Don Pedro, su hijo Francisco le sucedería en el cargo en 1734 (11). Siete años más tarde, otro de sus vástagos, Ignacio, compraría en juro de heredad la regiduría vacante por muerte de José Fernández de Mesa (12); siendo un hijo de Ignacio, también llamado Pedro, quien la herede en 1766 (13). Hallándose este último gravemente enfermo y sin sucesión, legó la plaza a su sobrina $\mathbf{M}^{\mathrm{a}}$ Josefa Bourgunyo en 1806, quien, al no poder ejercer el oficio por razón de su sexo, en 1814 elevó una petición a la Cámara de Castilla para nombrar teniente que la sustituyera en el Ayuntamiento (14).

Junto a la ostentación del dominio político, el disfrute del poder económico será otro de los rasgos comunes para este grupo de familias emparentadas, entre las que se encontraba la Casa de Bourgunyo.

A partir de los datos disponibles es posible conocer, de forma bastante aproxima$\mathrm{da}$, el patrimonio acumulado por este linaje así como los medios empleados para su consecución. A finales del siglo XVIII, el grueso de las propiedades de los Bourgunyo se centraba en una valiosa hacienda en la partida de la Condomina, la más rica de la huerta alicantina por los altos beneficios generados en la producción vinícola, y una residencia en las inmediaciones de la colegial de San Nicolás, en el centro de la ciudad. Pero cuando tuvo lugar el enlace entre Francisco y la primogénita de los Remiro Alvarez de Espejo, parte de los bienes de estos últimos pasaron a manos de la nueva familia de doña Francisca. Así, una vivienda en la capital, sita en el paseo de Ramiro al pie de las escalinatas de la iglesia de Santa María, y una hacienda en la huerta de Orihuela, término de la universidad de Almoradí, con su casa, ermita, bodega y tierras contiguas, engrosarán los bienes de los Bourgunyo a partir de 1650 (15).

Este pequeño núcleo en la Vega Baja pronto se vio incrementado. En las primeras décadas del setecientos el nuevo heredero de la familia, Pedro, contrajo matrimonio con Josefa Ruiz y Rocamora que, como avanzamos, pertenecía a una de las familias más distinguidas de la capital oriolana. La dote aportada por ésta consistió en 400 tahullas (16) de tierra-huerta (empleadas también en Almoradí) a las que pronto se le sumarían cerca de 180 tahullas más, mercadas por el regidor con ánimo de crear un 
vínculo en la zona (17). Igualmente, fruto de compras fueron las 220 hectáreas que componían la heredad de la Vallonga. Dicha partida, situada en el término de Alicante, resulta con mucho la más interesante de las propiedades acumuladas por la familia Bourgunyo. Desconocemos la fecha exacta de su adquisición, pero ya a partir de 1731 1732 sobresalía como una gran hacienda que comprendía la casa principal, una ermita, bodegas, cubas, lagares, balsas, etc., también destinada a la producción y elaboración de vino. Finalmente, y aunque de menor importancia, cabría citar dos fincas en las zonas de Carchofar y Sierra de Sancho, dedicadas a la misma especialidad (18).

Don Pedro falleció poco antes de iniciarse la década de los años cuarenta; pero, con anterioridad, ya había especificado su deseo de que la fortuna de los Bourgunyo se repartiese entre sus hijos Francisco e Ignacio con la debida igualdad, de modo que ninguno quedase perjudicado en el reparto de la herencia en beneficio del otro (19). Sobre Ignacio recayeron las posesiones de la Vallonga y tierras contiguas, así como algo más de 300 thas. en la huerta del Segura, mientras que Francisco, en calidad de primogénito, recibió el vínculo de la Condomina -al que se le habían añadido cerca de 70 tahullas en el Cabo de las Huertas- más el resto de las haciendas en la Vega Baja (20).

A juzgar por la documentación manejada, Ignacio decidió seguir los pasos de su progenitor dedicándose personalmente al cuidado de su patrimonio, incluso mejorándolo con sucesivas incorporaciones. Buena prueba de ello es que en el momento en que heredó la finca de la Vallonga, la producción vinícola se estimaba en torno a los 1.450 cántaros (21), manteniéndose cercana a los 3.000 durante todo el decenio. A partir de 1740 , la cifra ascendería a los 2.000 cántaros, duplicando esta cantidad diecisiete años después (22). Al margen de las compras efectuadas, las posesiones del segundogénito de los Bourgunyo se vieron reforzadas tras hacerse cargo, a mediados de siglo, del mayorazgo establecido por sus abuelos Miguel Ruiz y Luisa Rocamora (23). Debido a la escasa prole masculina y el temprano fallecimiento del mayor de los vástagos, los titulares decidieron que el mayorazgo recayera en el segundo hijo varón de cualquiera de sus tres hijas. La escasez de otros parientes próximos que pretendiesen la sucesión fue la causa de que Ignacio se beneficiara del citado vínculo. Sin embargo, hemos de resaltar la importancia de la claúsula de herencia: la necesidad de adoptar el apellido y armas de los Ruiz y Rocamora. Esta obligatoriedad, junto con la idea de que forzosamente heredase el segundo hijo varón se explicaría como una medida de prevención para que, en ningún caso, el fiduciario ostentara dos primogenituras al mismo tiempo, lo que podría hacer peligrar el núcleo patrimonial.

Como se ha señalado anteriormente, en 1744 Ignacio casó con Margarita Juan. Su madre, Violante Santacilia,pertenecía a una reputada familia ilicitana y su padre, Bernardo Juan, era Caballero de la Orden de San Juan, Gran Cruz y Baylio de Caspe y Comendador de Torrente y Mirambel (24). Desconocemos la cuantía de la dote aportada por Margarita, sin embargo, la suponemos elevada ya que la importancia del estamento nobiliario en la sociedad también se reflejaba en las altas cantidades que aportaban en este concepto (25).

El 13 de marzo de 1759, hallándose gravemente enfermo, Ignacio otorgó un poder 
a su mujer por el cual le facultaba ordenar su testamento. El mayor de sus ocho hijos, Pedro Bourgunyo y Juan, sería el legatario del mayorazgo Ruiz y Rocamora así como de la heredad de la Vallonga (26). Casi veinte años después -en diciembre de 1780- el titular, acogiéndose a un antiguo privilegio otorgado por Alfonso II en las cortes de Aragón de 1328, elevó una petición al monarca Carlos III para que se le concediese la jurisdicción alfonsina sobre esta última posesión, con el fin de crear en ella un lugar denominado Vallonga de Bourgunyo (27).

Por su parte, Francisco, el mayor de los herederos del regidor decano, contrajo matrimonio en dos ocasiones. Su primer enlace tuvo lugar en 1765; Juana Canicia, su mujer, era hija de Lorenza Pasqual de Ibarra, marquesa del Bosch, y Luis Rotla y Canicia, Caballero de la Orden de Montesa. Como bienes dotales, Juana aportó la cantidad de 1.424 libras, 9 sueldos y 4 dineros de moneda corriente, consistentes en dos capitales de censos, uno sobre la ciudad de Valencia y otro sobre los Propios del municipio alicantino (28). Pocos años después del fallecimiento de su primera consorte, Francisco se desposó nuevamente; esta vez con $\mathrm{M}^{\mathrm{a}}$ Antonia, hija de su fraterno Ignacio. Lo cierto es que esta unión resulta especialmente atractiva por representar el ejemplo más acabado de endogamia, a la par que ilustra la importancia de la política de alianzas de la élite que estudiamos. Por una parte, hemos de tener en cuenta que el enlace se producirá en unas fechas -1785- en que el patrimonio de Francisco había comenzado a experimentar gran menoscabo; en esta situación un nuevo matrimonio se presenta como clara alternativa para salvaguardar sus fragmentados bienes. La dote aportada por su sobrina, tanto en efectivo como en bienes muebles e inmuebles, ascendía a 11.918 libras, 8 sueldos y 3 dineros, cifra que, aunque nada despreciable, quedaba minimizada si se trataba, por un lado, de ayudar a levantar la economía del regidor $\mathrm{y}$, al mismo tiempo, favorecer un proceso de concentración patrimonial que respondía claramente a los ya decadentes intereses de la Casa de Bourgunyo (29). En este sentido, hemos de resaltar dos consideraciones expuestas por Chacón Jiménez en un reciente estudio sobre la familia española en la Edad Moderna. En primer lugar, la importancia que, durante estos siglos, jugó la endogamia como elemento corrector en las transmisiones hereditarias; idea que, al mismo tiempo, nos permitirá destacar el papel desempeñado por las mujeres en la enagenación del patrimonio familiar, sobre todo las pertenecientes a los grupos de poder que, a menudo, apartaban del nucleo original bienes que pasarían a manos distintas una vez que contrajeran matrimonio (30).

Finalmente, el último aspecto a enunciar es el relativo a la posesión de títulos de órdenes militares. Se ha verificado que los enlaces que analizamos van a suponer, al menos en tres casos, el emparentamiento entre familias ostentadoras de tal mención. Por lo que hace a la estirpe de los Canicia, habían estado ligados a la Orden de Montesa durante tres generaciones, amén de otros títulos y dignidades como la de familiares y alguaciles del Sa.to Oficio desde el siglo XVI (31). A esta misma institución pertenecieron dos miembros de la Casa de Bourgunyo: Ignacio y su hijo Pedro. El primero en calidad de familiar; el segundo como alguacil mayor del Tribunal en Alicante, en ausencia de su propietario Antonio Rotla y Canicia (32). Pero, al margen de estos datos, la importancia de este último radica en su adscripción a la Orden de 
Carlos III, de la que fue Caballero en el año 1790 (33). Otro de los hijos de Ignacio, Antonio, Capitán de Fragata de la Real Armada, obtendría el hábito de Nuestra Señora de Montesa por Decreto de 8 de agosto de 1795 (34). Por su parte, el grupo de los Juan se destacaron por su adscripción a la Orden de San Juan de Jerusalén, llegando a poseer, como en el caso de Bernardo, una encomienda.

Indudablemente, un análisis completo de la jerarquía que estudiamos requiere abordar tanto los aspectos relativos al nivel de vida que disfrutaban como al de sus relaciones sociales, mentalidad y costumbres. En este sentido es oportuna una descripción de la vivienda, su ordenación interior y utensilios contenidos, para ilustrar la potencialidad material y social que manifestaban los estamentos superiores.

Para la descripción del entorno inmediato de la Casa de Bourgunyo nos hemos servido de los inventarios post-obitum de Margarita Juan (35) y Francisco Bourgunyo (36), confeccionados en 1782 y 1797 respectivamente.

El regidor habitaba la vivienda familiar situada en el paseo de Ramiro. Desgraciadamente, la descripción notarial no señala algunas de las características de la casa tales como el número de plantas o el de habitaciones; pero, a juzgar por el mobiliario descrito, suponemos una residencia de grandes dimensiones. Presumiblemente, la planta baja estaría destinada a la cocina y dependencias anexas. Sin embargo, en contra de lo tradicionalmente establecido, se revelan como estancias muy amplias en atención al menaje descrito: cazuelas, ollas, cucharones, pescaderas, chocolateras, cafeteras, sartenes, escudillas, artesas, homos etc...

Por otra parte, suponemos la existencia de gran número de dormitorios ya que hemos cuantificado hasta treinta y dos tablados de cama -con sus respectivas cabeceras-, dos canapés, dos catres y veinticuatro colchones. Tanto las alcobas como las restantes estancias estarían ricamente ornamentadas y decoradas con una variada gama de cortinajes y tapices, arcas, arcones, tocadores, biombos, armarios, baules, cofres, espejos, colchas de tafetán liso o de colores y almohadas de respaldo de damasco carmesí. La cantidad de sillas inventariadas -hasta ciento treinta y dos- en diferentes tapizados y maderas (nogal, roble, pino o morera) indica que la vivienda estaría dotada de varias salas, salitas, comedores y, al menos, un despacho; engalanados con cuadros, alfombras, cortinas, rinconeras para la cristalería, candelabros, “... un escritorio charolado con pies de cabra", aparadores y piezas similares donde se contenían servilletas, manteles y paños en diferentes telas y/o encajes. Asimismo, el cuidado que la familia prodigaba al ocio queda recogido en los inventarios. Existen diversas disposiciones que indicaban la necesidad de pagar deudas atrasadas al profesor de música de Lorenza, hija de Francisco; la mención a varios instrumentos musicales como dos flautas y un clave; o la aparición de pasatiempos como el chaquet o las damas. También las labores de costura estarían al orden del día: un tambor de bordar, dos tornitos de hilar, una debanadera, agujas de plata y cerca de un centenar de varas de tela para la confección de cortinas, sábanas o manteles así lo demuestran.

El nivel de vida queda mejor expresado en informaciones relativas a la cuantificación de piezas de platería encontradas en los hogares. En este sentido, el inventario de 
Margarita Juan nos ofrece una muestra bastante representativa: docenas de platos, cuadrados y ovalados, en diferentes tamaños; salvillas, bandejas, ensaladeras, cafeteras, jarrones, portaretratos o palilleros, además de todo tipo de cubertería. Tampoco podemos dejar de mencionar el vestuario o las joyas tasadas, altamente representativas de la importancia concedida a las apariencias externas como valores sociales. El capítulo de la indumentaria está representado ejemplarmente en la cantidad y calidad: profusión de chaquetas, batas de satén, ratina o bombasí; calzones de paño o lienzo; capas, uniformes de verano, camisas, calcetas; jubones, justillos, decenas de vestidos de tafetán, seda, algodón o lino; mantos, mantillas florentinas, etc... Por lo que hace a la joyería, merecen destacarse: sortijas de diamantes, varios juegos de hebillas en oro y plata, algunas veneras de la Inquisición, una cruz de oro con esmeraldas, tumbagas y tumbagones también con esmeraldas, un camafeo guarnecido con diamantes y rubíes o "... unos brazaletes con perlas finas y menudas".

El fasto consumista de que hacía uso la nobleza influyó, en ocasiones, en su vertiginosa decadencia, ya que, como hemos visto, se necesitaba un considerable capital para afrontar el estilo de vida que obligaba su categoría. Decidida a mantener este nivel, más de una familia tuvo que recurrir a la petición de préstamos como medida "razonable" para afrontar los extraordinarios gastos (37). En este sentido, el inventario de bienes realizado tras la muerte de Francisco Bourgunyo refleja su deteriorada situación económica a tenor de las tierras hipotecadas, ventas o la cantidad de deudas acumuladas a sirvientes, comerciantes, panaderos, tenderos, médico, etc...

Lo cierto es que esta "delicada" situación económica no era nueva para el regidor. Apenas tres años después de contraer matrimonio, Juana Canicia emitió duros reproches contra su marido, alegando que la causa de estas pérdidas estaba motivada por su afición al juego:

“... Lo que más sensible se le haze, ocacionandole la mayor pena, es que procurando la suplicante el más retirado modo de vivir sin gastos de visitas, trato con nadie, galas, ni otro tren de vida con el que se mantuvo en casa de sus padres, sólo por el extraño genio de su marido (...) por más que la suplicante procura reducirse, se esmera mas aquel en jugar continuamente sin poder conservar para mantener sus obligaciones por su mala conducta, causa pricipal de los atrazos que sostiene en bien cerca de nueve mil pesos que está deviendo, sin embargo de que la pingue renta del mayorazgo, bienes libres, sueldo de Teniente Coronel retirado y el de Regidor de dicha ciudad le producen anualmente cerca de unos quatro mil (...) " (38).

Pero, a decir de doña Juana, las quejas contra el regidor tenían fundadas razones ya que su esposo, acosado por los acreedores y resentido de las pérdidas, solía arremeter contra ella y la hija de ambos propinándoles no sólo graves insultos sino que “... en ciertas ocaciones la amenazaba de obra con accion de levantar un palo que acostumbraba usar". En el relato de toda la serie de ultrajes y mortificaciones que padecía, la exponiente incluye que en cierta ocasión tuvo que acudir en su ayuda el vicario foráneo ya que 
“... no satisfecho con semejante antecedente y cerrandola con violencia en un quarto en su compañia, reiteró su furia haziendola del cuello y tapandole la boca en terminos de quererla aogar, como tal vez lo uviera conseguido si â los clamores y lloros de la lastimada pidiendo Justicia âl Cielo, no se uviera alborotado la Calle acudiendo Gentes que compadecidas lo pusieron en noticia del Vicario Foraneo â instancias de la suplicante (...)".

Tras abandonar el hogar conyugal marchándose a la casa paterna, Juana puso en conocimiento de las autoridades su situación solicitando que le fuera concedida la demanda de divorcio contra su marido. Sin embargo, ésta no llegó a prosperar; tanto las autoridades civiles como eclesiásticas consideraron la queja "... frívola y excesiva " ordenándole guardar el justo respeto y obediencia a su esposo, y que en lo sucesivo ambos mantuvieran la sociedad y armonía propias de su estado.

Si las relaciones de Francisco con su primera mujer no fueron del todo amistosas, tampoco parece que lo fueran con la hija de ambos. Así se desprende de un análisis del testamento del citado Bourgunyo, redactado en treinta de noviembre de 1793 (39). Por el mismo, el titular impelaba a su hija y yerno a una "... final reconcialiación y amistosa transacción (...) “, tras doce años de litigios en razón de la legítima que pertenecía a esta última por el fallecimento de su madre. A juzgar por información recogida, estas no fueron las únicas ocasiones en que las mujeres de la Casa de los Canicia manifestaban un " cierto carácter temperamental"; en este sentido, Giménez López relata ciertos problemas surgidos, por diferencias de trato, entre las féminas de esta poderosa familia con el corregidor teniente general Marqués de Alós en 1758. El conflicto estalló cuando Mariana Vaillo de Llanos y Lorenza Pasqual de Ibarra, esposa y madre respectivamente de Antonio Canicia, negaron el tratamiento de Excelencia al recién ascendido teniente general. Lo que en principio parecía mera cuestión protocolaria, derivó en un grave problema político en el que tuvieron que intervenir instituciones superiores (40).

Por último, consideramos conveniente examinar uno de los temas más interesantes en relación a la "mentalidad" demostrada por la élite que estudiamos: sus posiciones ante la religión y la muerte. La información extraída de los citados inventarios, aunque cuantitativamente importante, resulta en ocasiones algo contradictoria. Por un lado se describen toda la serie de rosarios, escapularios y relicarios tan comunes en la época (un San Miguel en plata, varios rosarios en nacar y cadenas de oro de diferentes advocaciones ) así como una amplia muestra de cuadros de contenido religioso ( uno de la Virgen de Belén, dos de la Sagrada familia, un Descendimiento, un Ecce Homo, otro de San Antonio de Padua, etc...). Pero llama la atención la escasa representación de libros dedicados a esta materia, limitándose, para Margarita Juan, a "Verdades católicas "; " Año Virgines "; y un tercero de San Luis Gonzaga. En relación a la muerte, y siguiendo con los usos establecidos, los testamentos reflejan emociones piadosas y la inequívoca profesión de fe junto a las tradicionales mandas para obras de caridad y sufragio de almas. De este modo resulta frecuente que se dispusiesen determinadas cantidades en metálico para conventos y hospitales de la localidad así como el deseo de que a su muerte se mandesen decir cientos - incluso miles - de misas cantadas o rezadas. Estas peticiones normalmente iban acompañadas por una serie de indicaciones 
sobre el hábito que deseaban vestir: capuchinas, carmelitas, dominicos ...; el número de cirios, velas o candelas con que iluminar la iglesia y el lugar donde debían ser enterrados. A modo de ejemplo, Margarita Juan decidió que le dieran sepultura junto a su padre, Bernardo (41), mientras que su hija $\mathrm{M}^{\mathrm{a}}$ Antonia Bourgunyo pidió ser inhumada en la parroquial de Santa María de Alicante, en el panteón de los marqueses del Bosch, donde yacía su marido (42).

\section{NOTAS}

1.- Chacón Jiménez, F. Historia social de la familia en España. Alicante, 1990.

2.- A.H.N Estado, Indice de pruebas de Carlos III. Expediente de Pedro Bourgunyo y Juan. Leg 460 .

3.- Entre las crónicas y nobiliarios consultados figuran:

- Viravens Pastor, R. Crónica de la Muy Ilustre y Siempre Fiel Ciudad de Alicante. Alicante, 1989. Reed. Facsimil a cargo del Ayuntamiento de Alicante - Banco de Alicante.

- Arques Jover. Nobiliario Alicantino. Trans. y notas de Luis Mas y Gil y J.M. Esquerdo. Alicante, 1966

- Orts y Bosch, P. Alicante: notas históricas. 1373-1800. Valencia, 1971.

- Viciana, M. Chrónica de la Inclita y Coronada Ciudad de Valencia y su Reyno. Reed. facsimil a cargo del Dpto. de Historia Moderna de la Universidad de Valencia. Valencia, 197273.

- Bendicho, V. Chrónica de la M.I Noble y Leal ciudad de Alicante, año 1640. Manuscrito conservado en al Archivo Municipal de Alicante (en adelante A.M.A ).

4.-_Privilegios y Provisiones reales. Arm 1, Lib 54, Fol 101 y ss.

5.- Mas y Gil,L." El Archivo Municipal y sus registros sobre linajes ", en Hidalguía n" 43, pag $819-832$.

6.- A.H.N Estado, Pruebas de Carlos III, Op. Cit fol 113.

7.- El Archivo Municipal de Alicante ha sufrido constantes alteraciones en su composición a consecuencia del bombardeo francés de 1691. Durante la guerra de Sucesión parte de los fondos, semidestruídos, fueron trasladados a Mallorca para su mejor custodia; volviendo, años después, los escasos documentos que se salvaron de la quema y los desatres.

8.- A.M.A, Arm 1, lib 17, fol 76 y Lib 22, fol 44 vto.

9.- La relación de oficios ejercidos por la familia Bourgunyo durante época foral ha sido elaborado a partir de las fuentes siguientes:

- Arques Jover, A. Op. Cit pag 121-162

- A.H.N. Estado. Indice de pruebas... Op. Cit, fols 113- 117 vto.

- A.M.A.Indice de personas que sirvieron en cargos publicos, S/c.

- Alberola Romá. A. Jurisdicción y propiedad de la tierra en Alicante. siglos XVII-XVIII. Alicante, 1984. Pag 118-128. 
10.- A.M.A, Arm 1, Lib 25.

11.- A.M.A, Arm 9, Lib 27, Fol 140-143 vto.

12.- A.H.N Consejos, Memorial de Ignacio Bourgunyo solicitando plaza de regidor por el estado noble, Leg. 18.333.

13.- A.M.A Arm 12, Lib 16, fol 173.

14.- A.H.N Consejos, Permiso para que $\mathbf{M}^{\mathbf{a}}$ Josefa Bourgunyo nombre teniente en el Ayuntamiento. Leg 18.335

15.- A.H.N. Estado. Pruebas de ... Op.Cit Fol 66- 66 vto.

16.- una tahulla de tierra equivale a 0'1201 áreas.

17.- A.H.N. Estado. Pruebas de Carlos III... Op. Cit fol 104 y ss.

18.- A.M.A. Arm 5, Lib 113. Justiprecio general del Término. Año 1756.

19.- A.H.N. Estado. Pruebas... Op. Cit, testamento de Pedro Bourgunyoy Remiro. fol. 106 vto-107

20.- Ibidem

21.- un cántaro de vino equivale a 11'5 litros.

22.- A.M.A, Arm 17 S/n. Libros del manifiesto del vino. Años 1709-1799. Volúmenes correspondientes a los años consignados.

23.- A.H.N. Estado. Pruebas... Op. Cit, testamento Miguel Ruiz - Luisa Rocamora; fol 5156

24.- Ibid. fol 111-112 vto.

25.- Castaño Díaz, J.A. Los contratos matrimoniales en el Antiguo Régimen. 1750-1775. Memoria de licenciatura inédita. Universidad de Alicante, 1983.

26.- A.H.N Estado. Pruebas..., Op. Cit, fol $70-83$ vto.

27.- A.M.A Arm 1, Lib 60, Fol 329-375

28.- A.H.P.A, Testamento de Francisco Bourgunyo. P/ 1242.

29.- Ibidem

30.- Chacón Jimenez, Op. Cit Pag 27.

31.- Barón de Finestrat. Nobiliario Alicantino. Alicante, 1983. Pag 97 - 101

32.- A.M.A, Arm 1. Lib 68, fol 40.

33.- A.H.N.Estado. Pruebas. Expediente completo donde se contienen las pruebas presentadas por Pedro Bourgunyo y Juan y su nombramiento como Caballero.

34.- A.H.N Expedientillos. Ordenes militares. Leg 183. № $156 \mathrm{~S} / \mathrm{F}$.

35.- A.H.P.A, Partición y Diivisión de bienes de Margarita Juan. año 1782. P/ 1452

36.- A.H.P.A Partición y división de bienes de Francisco Bourgunyo. año 1797. P/85.

37.- Stone, L. La crisis de la aristocracia. 1558-1641. Madrid, 1985.

38.- A.G.S. Guerra Moderna. Leg, 1.380, Discordias en el matrimonio Bourgunyo-Canicia.

39.- A.H.P.A. P/ 1242.

40.- Giménez López, E. Militares en Valencia. 1707-1808. Alicante, 1990. pág 3 a 8.

41.- A.H.P.A. Testamento de Margarita Juan. P/1450. Año 1774.

42.- A.H.P.A. Testamento de $\mathrm{M}^{\mathrm{a}}$ Antonia Bourgunyo. P/90. Año 1801. 


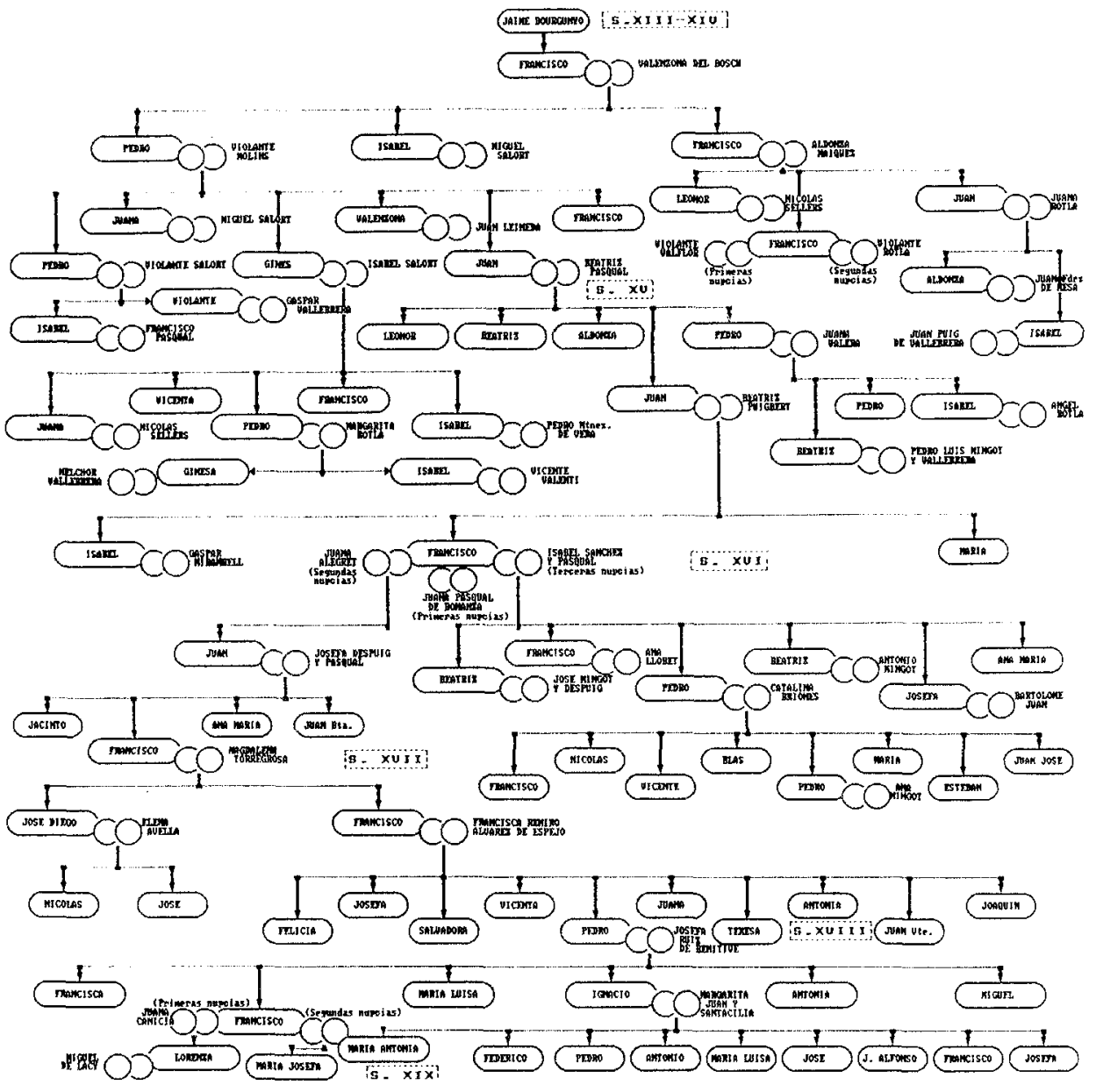

CUADRO GENEALOGCO DE LA

FAMILTA BOURGUNYO. STGLOS XII - XVIII 


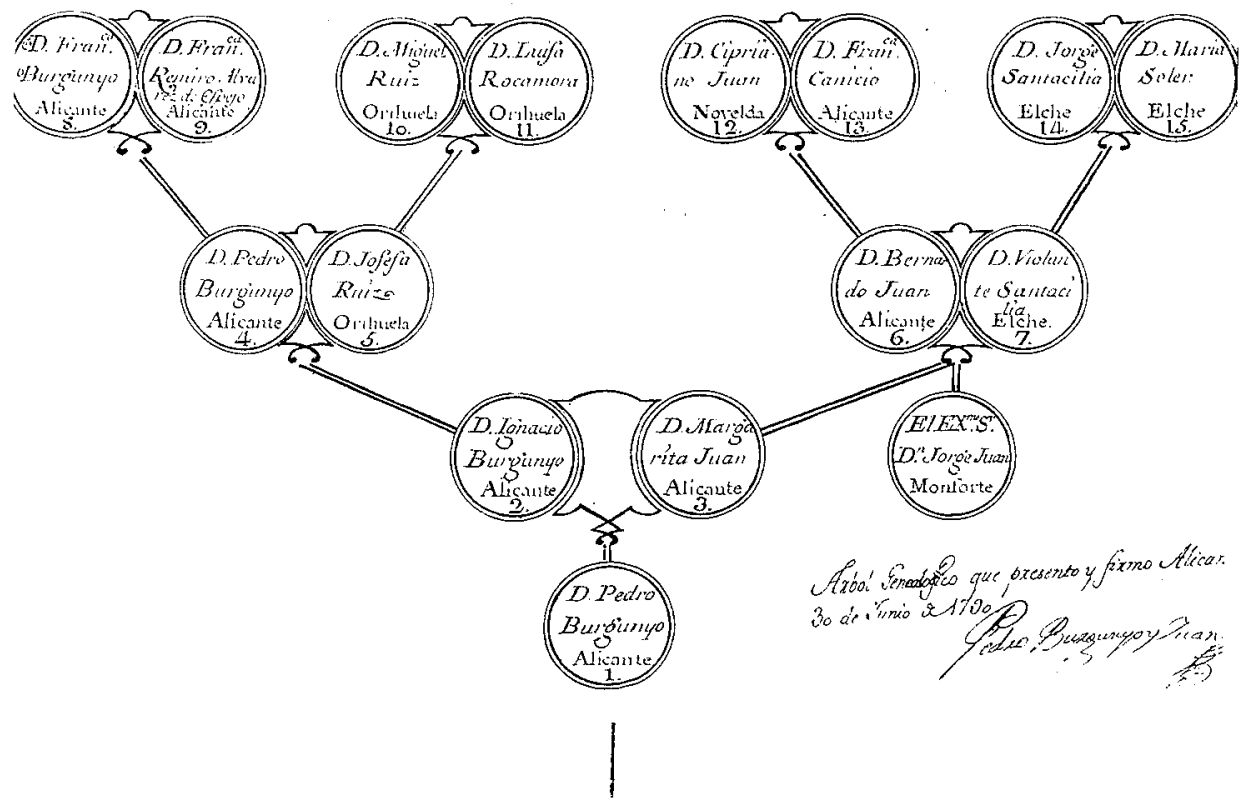

